\title{
Effect of local treatment with adipose tissue-derived mesenchymal stem cells in the early tumorigenesis of osteosarcoma
}

\author{
SHEEN-WOO LEE ${ }^{1}$, TAE JOO JEON ${ }^{2}$ and SANDIP BISWAL ${ }^{3}$ \\ ${ }^{1}$ Department of Radiology, Gil Hospital, Gachon University School of Medicine and Science, Incheon 406-799; \\ ${ }^{2}$ Department of Nuclear Medicine, Gangnam Severance Hospital, Yonsei University \\ College of Medicine, Seoul 135-720, Republic of Korea; ${ }^{3}$ Department of Radiology, \\ Stanford University School of Medicine, Stanford, CA 94305-5105, USA
}

Received October 22, 2014; Accepted December 12, 2014

DOI: $10.3892 /$ or.2015.3711

\begin{abstract}
There are conflicting data describing the effect of mesenchymal stem cells (MSCs) on tumorigenesis. The present study aimed to determine the survival rate and effect of adipose tissue-derived MSCs (ADMSCs) in tumor growth using bioluminescence imaging (BLI) and ultrasound (US) in an osteosarcoma xenograft model. Firefly luciferaseexpressing ADMSCs combined with the osteosarcoma cell line UMR-106 in 4 different proportions (5, 10, 15 and 25\%, named G1-G4, respectively) were xenografted into the right flanks of nude mice. The same number of UMR-106 cells was inoculated into the contralateral side of each mouse. Serial bioluminescence images were captured over 16 days to monitor the presence of ADMSCs in each group of 5 animals. The tumor volume was measured by ultra-high resolution US, and the tumor volume ratio (AMDSC mixed xenograft/ control xenograft) was obtained to evaluate the effect of AMDSCs on tumor growth. Immunohistochemistry was performed to confirm the distribution of residual AMDSCs in the tumor. In G1, G2 and G3, the suppression of tumor growth by AMDSCs was noted in 2/5, 4/5 and 4/5 mice, respectively. However, accelerated tumor growth was noted in G4, which had the highest proportion of ADMSCs. The tumor volume ratio was significantly lower in G2 and G3 compared to $\mathrm{G} 4$, by Mann-Whitney $\mathrm{U}$ test $(\mathrm{P}=0.0159)$. Bioluminescence images demonstrated a serial decrement of the reporter gene for ADMSCs in the tumor mass without evidence of proliferation. Immunohistochemistry staining revealed minimal residual ADMSCs in the tumor periphery. Taken together, our data revealed that direct inoculation of ADMSCs into a tumor xenograft caused the death of the majority of ADMSCs in the tumor mass. Furthermore, relatively low proportions
\end{abstract}

Correspondence to: Dr Tae Joo Jeon, Department of Nuclear Medicine, Yonsei University College of Medicine, 712 Eonju-ro, Kangnam-gu, Seoul 135-720, Republic of Korea

E-mail: tjeonnm@yuhs.ac

Key words: mesenchymal stem cells, adipose-derived stem cells, tumors, molecular imaging of ADMSCs suppressed the growth of osteosarcoma, while higher proportions showed a tumor-promoting effect.

\section{Introduction}

Since the first embryonic stem cell (ESC) line derived from human blastocysts was described by Thomson in 1998 (1), ESCs have received much attention for their great potential in medicine. However, ethical issues related to ESCs are a major obstacle to their application in clinical practice (2), and therefore, adult stem cells were suggested as an alternative (3). Mesenchymal stem cells (MSCs) are one such candidates, and have been widely investigated due to their potential application in the treatment of chronic wounds, metabolic bone disease, fractures and myocardial infarction (4). Furthermore, the diverse effects of these cells on cancer highlight their possible application in cancer treatment (5-10). However, there have been many contradictory reports concerning the effects of MSCs in tumorigenesis, including the promotion (11-13) and inhibition (14) of tumor growth.

To date, most cancer-related studies have been performed using bone marrow-derived mesenchymal stem cells (BMMSCs) $(6-8,15,16)$, while studies using adipose tissuederived ADMSCs are rare. This is despite the many advantages of using ADMSCs, which include easier harvest, higher yield and comparable differentiation potential compared to BMMSCs (17). In addition, most studies concerning the tumorigenesis of ADMSCs have been performed after the intravenous injection of cells, i.e., systemic administration (7), and have involved in vitro experiments (18). To the best of our knowledge, there is no in vivo study dealing with the effect and behavior of locally administered ADMSCs in early tumor formation.

Recent advances in bioluminescence imaging (BLI) techniques have enabled us to assess the status of cells administered to living animals without sacrifice during the follow-up period. Furthermore, high resolution ultrasound (US) has made it possible to precisely measure small objects by enabling the volumetric calculation of the tumor mass in various shapes and locations. In the present study, we aimed to evaluate the proliferation and survival of local treatment with ADMSCs in early tumor formation by monitoring the reporter ADMSCs 
using BLI. We also evaluated the effect of these cells on tumor growth by the comparison of serial BLI data and the tumor volume measured by small animal-dedicated high resolution US.

\section{Materials and methods}

Tumor cells. UMR-106 osteosarcoma cells (UMRs), which are a clonal derivative of a transplantable rat osteosarcoma induced by the injection of radiophosphorus, were purchased from the American Type Culture Collection (ATCC; Manassas, VA, USA). The cells were cultured in Dulbecco's modified Eagle's medium (DMEM) containing 10\% fetal bovine serum (FBS) and $100 \mathrm{IU} / \mathrm{ml}$ penicillin, $100 \mathrm{IU} / \mathrm{ml}$ streptomycin and $0.25 \mu \mathrm{g} /$ $\mathrm{ml}$ amphotericin at $37^{\circ} \mathrm{C}$ in an atmosphere of $5 \% \mathrm{CO}_{2}$, and were passaged by standard methods of trypsinization. Only cells at passages 2 and 3 were used in the experiments.

Mesenchymal stem cell harvest and culture. All experiments were performed following animal protocols approved by the Institutional Animal Care and Use Committee at Stanford University. ADMSCs with permanent expression of a reporter gene signal during follow-up BLI were extracted from the adipose tissues of transgenic mice expressing the $\beta$-actin promoter and double reporter genes of GFP and firefly luciferase. This was performed in order to minimize errors in BLI data caused by the viral transfection of reporter genes into ADMSCs. The transgenic mice used for the harvesting of ADMSCs were kindly supplied by Dr Contag's laboratory at Stanford University. Subcutaneous fat tissues were collected from the lower anterior abdominal wall to the inguinal area of the transgenic mice. The tissue fragments were rinsed 3 times in phosphate-buffered saline (PBS) before being finely minced for $5 \mathrm{~min}$. These tissues were then digested using $0.075 \%$ collagenase (Sigma-Aldrich, St. Louis, MO, USA) at $37^{\circ} \mathrm{C}$ for $1 \mathrm{~h}$. Neutralized cells were centrifuged and the mature adipocytes and fibrovascular fraction were selected and removed. Pelleted stromal cells were passed through a $100-\mu \mathrm{m}$ cell strainer before plating. Cells were cultured at $37^{\circ} \mathrm{C}$ in an atmosphere of $5 \%$ $\mathrm{CO}_{2}$, in DMEM containing 10\% FBS, $100 \mathrm{IU} / \mathrm{ml}$ penicillin, $100 \mathrm{IU} / \mathrm{ml}$ streptomycin and $0.25 \mu \mathrm{g} / \mathrm{ml}$ amphotericin. Only the cells that adhered to plastic were used in this experiment, and cells floating during culture were discarded. Preliminary in vitro BLI of cultured ADMSCs was performed to validate the performance of luciferase gene expression before implantation in animals.

Local administration of ADMSCs in an osteosarcoma xenograft model. Twenty nude mice (NU/NU) aged 8-10 weeks were purchased from Charles River Laboratories (Wilmington, MA, USA) and categorized into 4 groups. Each group was injected with ADMSC-UMR mixtures containing 5, 10, 15, or 25\% ADMSCs, defined as G1-G4, respectively. This experiment was performed twice separately due to limitations in stem cell harvest and culture, the preparation of UMRs and BLI and US imaging capacity.

Different numbers of ADMSCs and UMRs were mixed with $30 \mu \mathrm{l}$ of solubilized basement membrane preparation without growth factor (Matrigel; BD Biosciences, San Jose, CA, USA) and subcutaneously injected into the right flank of
Table I. Number of UMR-106 cells and ADMSCs.

\begin{tabular}{lcrc}
\hline Group & UMR-106 cells & ADMSCs & Proportion (\%) \\
\hline G1 & $1,000,000$ & 50,000 & 5 \\
G2 & $2,000,000$ & 200,000 & 10 \\
G3 & $1,000,000$ & 150,000 & 15 \\
G4 & $2,000,000$ & 500,000 & 25 \\
\hline
\end{tabular}

the mice. The tumor xenograft was performed in 4 groups at 2 different time points. The first 2 groups, G1 and G3, were injected with mixtures of $5 \times 10^{4}$ and $1.5 \times 10^{5}$ ADMSCs in $1 \times 10^{6}$ UMRs. The second two groups, G2 and G4, were injected with $2 \times 10^{5}$ and $5 \times 10^{5}$ ADMSCs in $2 \times 10^{6}$ UMRs. As a control, $1 \times 10^{6}$ and $2 \times 10^{6}$ UMRs in basement membrane preparation were xenografted at the contralateral area of mice in the first (G1 and G3) and second groups (G2 and G4), respectively. The numbers of ADMSCs and UMRs and proportions of ADMSCs in each group are summarized in Table I. These procedures were carried out under general anesthesia with isoflurane inhalation.

Tumor volume obtained by small animal-dedicated high resolution ultrasound. A specially designed high-resolution micro-US system for small animal with a $40 \mathrm{MHz}$ transducer (Vevo 770; Visual Sonics, Toronto, Ontario, Canada) was used to evaluate the characteristics of the growing tumor implants with and without ADMSCs. The spatial resolution and penetration depth of the $40 \mathrm{MHz}$ transducer used in this study was $30 \mu \mathrm{m}$ and $6 \mathrm{~mm}$, respectively. US imaging was performed at 2- or 3-day intervals before sacrificing the animals 16 days after the xenograft. In order to obtain the exact tumor volume taking into account various contours, we assumed each tumor mass was an elliptical ball and obtained two tangential US images along the longitudinal and transverse planes to calculate the exact volume. The equation for the calculation of this mass is as follows: Volume $=4 / 3 \pi \mathrm{ABC}$, where A, B and C are the measured radii of each elliptical ball, and the unit of the calculated volume was $\mathrm{mm}^{3}$ (Fig. 1).

The changes in the tumor volumes of the ADMSC-treated and control sides were monitored and compared in each group. The ratio of tumor volume (volume of ADMSC-treated site/ volume of control side) was also measured for each mouse, and these results were compared between the groups to reduce the bias from individual variance. The tumor-suppressive or tumor-promoting effect of ADMSCs was determined by the comparison of the ratios of tumor volume on the final day as well as the mean volumes.

In vivo BLI. An $\sim 30 \mathrm{mg} / \mathrm{ml}$ solution of luciferin was made by dissolving $1 \mathrm{~g}$ D-luciferin firefly potassium salt (Biosynth International, IL, USA) in $33.3 \mathrm{ml}$ of PBS. Approximately $4.5 \mathrm{mg} / 150 \mu \mathrm{l}$ of luciferin was injected into the intraperitoneal space of each mouse. BLI was obtained 10 min after the administration of luciferin using a cooled charge-coupled device camera (IVIS; Xenogen). Mice were imaged at high resolution for $5 \mathrm{~min}$, and five mice were simultaneously imaged in the prone position. BLIs were performed for 16 days 

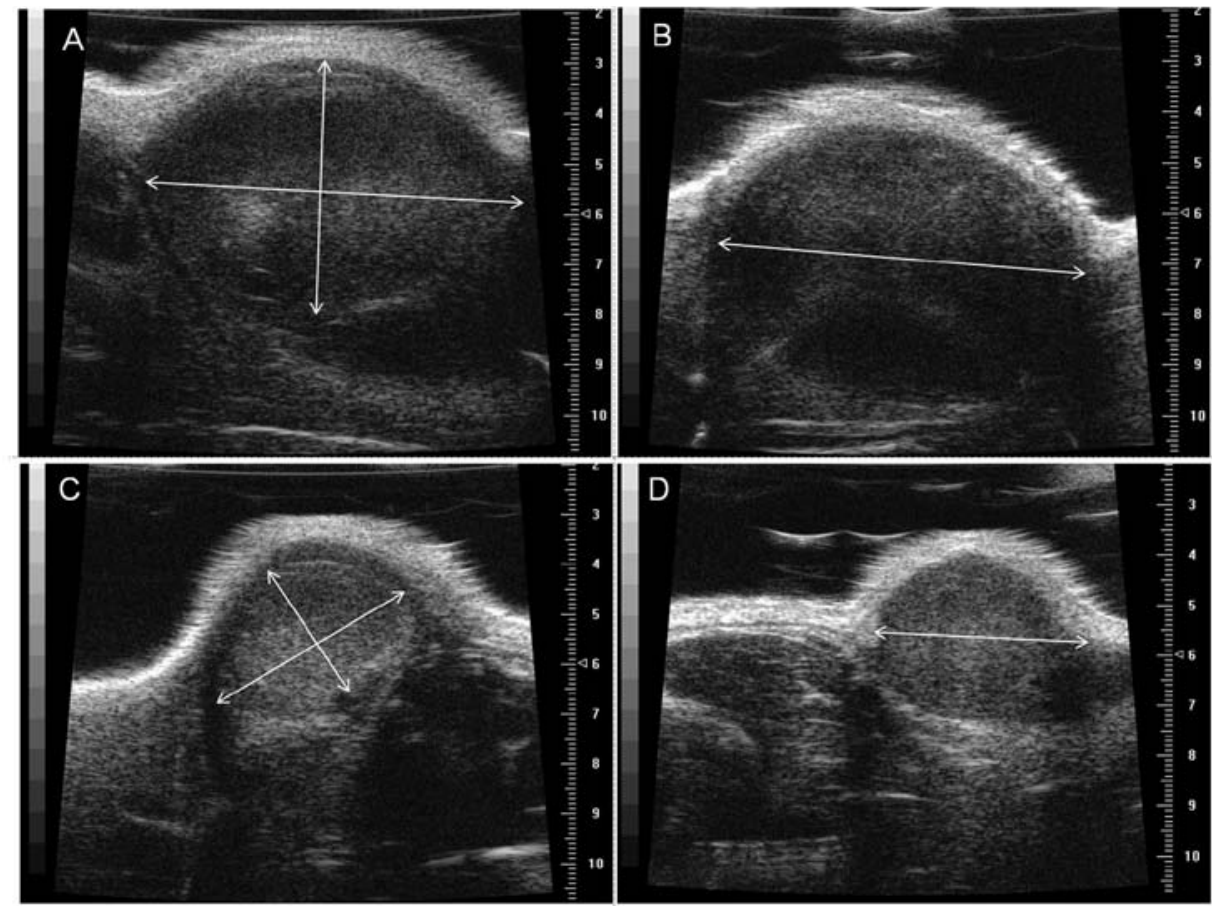

Figure 1. Assessment of tumor volume by ultra-high resolution ultrasound. Ultra-high resolution US images of targeted tumors were obtained in both the longitudinal and transverse directions. Three radii of each mass at $90^{\circ}$ angles were measured to calculate the tumor volume. The control tumor revealed larger volume by longitudinal (A) and transverse (B) measurement, compared with the contralateral side treated with stem cells (C, D).

with 2-day intervals for G1 and G3, and 3-day intervals for G2 and G4. The acquired BLI data were analyzed by a specialized program (Living Image Software; Xenogen USA). A round region of interest with a $3.2-\mathrm{cm}$ diameter was located in the ADMSC-inoculated right flank to measure the luciferase activity from the ADMSCs. The measured bioluminescence activity was expressed as average radiance (photon $/ \mathrm{cm}^{2} / \mathrm{sec} /$ steradian).

Immunohistochemistry. The rabbit anti-firefly luciferase antibody was obtained from Abcam (cat. no. ab21176, Cambridge, UK). Xenografts were embedded in optimum cutting temperature compound (Sakura Finetek, Torrance, CA, USA) using liquid nitrogen. Sections of $10-\mu \mathrm{m}$ thickness were fixed in $2 \%$ paraformaldehyde for $10 \mathrm{~min}$, and then washed using PBS. Prior to incubation with the antibodies, the sections were blocked using PBS containing $0.1 \%$ Triton $\mathrm{X}-100$ and $5 \%$ normal goat serum. The primary antibody was diluted (1:50) in blocking solution and applied overnight at $4^{\circ} \mathrm{C}$. Alexa 488-conjugated goat anti-rabbit IgG secondary antibody (Invitrogen, Carlsbad, CA, USA) was then added at a 1:100 dilution for $30 \mathrm{~min}$ at room temperature. Stained sections were mounted using antifade reagent containing 4',6-diamidino-2-phenylindole dihydrochloride (DAPI; Vecta Shield, Burlingame, CA, USA) to visualize cell nuclei. Fluorescent microscopic images were captured using the Nikon Eclipse E800 (Nikon Imaging).

Statistical analysis. The mean volumes of the xenografted tumors on the ADMSC-treated UMR side (right) and the UMR-only control side were compared using the Wilcoxon matched-pairs signed ranks test to determine the differences in tumor volumes in the same subjects. Then the volume ratio of the ADMSC-treated UMR mass to the control tumor was calculated in each mouse from the same group, and the means of these ratios were compared across groups using the MannWhitney U test.

The Pearson correlation coefficient was used to evaluate the correlation between BLI activity and tumor volume in G2 and $\mathrm{G} 3 . \mathrm{P}<0.05$ was taken to indicate a statistically significant difference. All statistical evaluations were performed using SAS statistical software (ver. 9.2; SAS Institute Inc., Cary, NC, USA).

\section{Results}

Comparison of the changes in tumor volumes and proportions of ADMSCs. The initial volume of injection on both sides of each mouse was $30 \mathrm{~mm}^{3}$ in all 20 mice in the 4 groups. On postoperation date (POD)2, the volume of xenografts was slightly decreased compared with the initial measurement time due to the absorption of water from the basement membrane preparation used. Thereafter, the volume of the tumors continuously increased on both the ADMSC-treated and control sides, but the increment pattern was different among all 4 groups. In the group containing the lowest proportion of ADMSCs, G1 (5\%), the ADMSC-treated xenograft was smaller than the contralateral tumor-only side in $2 / 5$ mice. The remaining 3 mice had a larger tumor volume on the ADMSC-treated side. The injection of mice with higher proportions of ADMSCs (G2 and G3, 10 and $15 \%$, respectively) led to the development of smaller tumors in 4/5 mice (Fig. 2). However, all mice in the group with the highest proportion of ADMSCs, group G4 (25\%), showed larger tumor volumes. These results are summarized in Table II. The Wilcoxon matched-pairs signed-ranks test revealed no statistically significant difference in the mean 
Table II. Average volume of the xenografted tumors in each group.

\begin{tabular}{|c|c|c|c|c|c|c|c|}
\hline & & POD2 & POD5 & POD8 & POD11 & POD13 & POD16 \\
\hline \multirow[t]{2}{*}{ G1 } & ADMSCs & 7.19 & 12.44 & 13.37 & 39.72 & 53.80 & 72.91 \\
\hline & Control & 7.93 & 4.84 & 6.01 & 10.35 & 19.14 & 38.06 \\
\hline \multirow[t]{2}{*}{ G2 } & ADMSCs & 20.04 & 18.31 & 35.08 & 69.59 & 128.32 & 144.61 \\
\hline & Control & 30.06 & 58.14 & 110.55 & 170.42 & 234.18 & 308.23 \\
\hline \multirow[t]{2}{*}{ G3 } & ADMSCs & 6.81 & 9.91 & 12.35 & 17.24 & 74.96 & 93.39 \\
\hline & Control & 7.60 & 6.12 & 7.23 & 16.35 & 75.86 & 127.56 \\
\hline \multirow[t]{2}{*}{ G4 } & ADMSCs & 26.23 & 27.71 & 56.72 & 78.67 & 133.58 & 693.43 \\
\hline & Control & 27.54 & 14.66 & 19.18 & 46.43 & 65.03 & 259.84 \\
\hline
\end{tabular}

POD, post-operation date; ADMSCs, adipose tissue-derived mesenchymal stem cells.

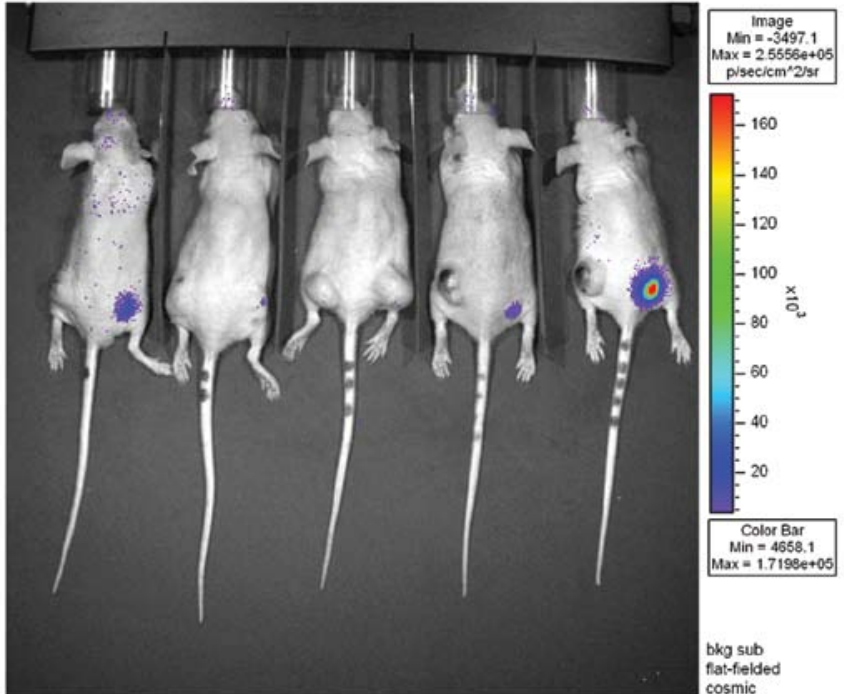

Figure 2. Detection of ADMSC-mediated tumor suppression by BLI. Upon BLI of G2 on the final day, all mice but one (\#3) revealed suppressed tumor growth in the ADMSC-treated UMR xenografts (right flank of the mice) compared with UMR-only masses on the contralateral side.

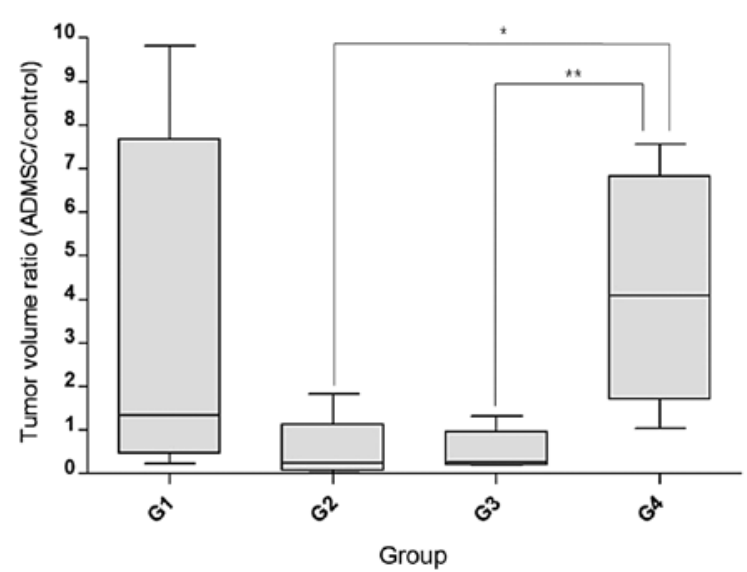

Figure 3. Assessment of tumor suppression by ADMSCs using tumor volume ratios. The mean tumor volume ratios (ADMSC-UMR xenograft/UMR-only xenograft) in G2 and G3 were $<1$, showing a tumor-suppressive effect of AMDSCs, while the ratio in G4 was $>1$, showing a tumor-promoting effect of ADMSCs. The P-value of the Mann-Whitney U test between *G2 vs. G4, and ${ }^{* *} \mathrm{G} 3$ vs. G4 was $<0.05$

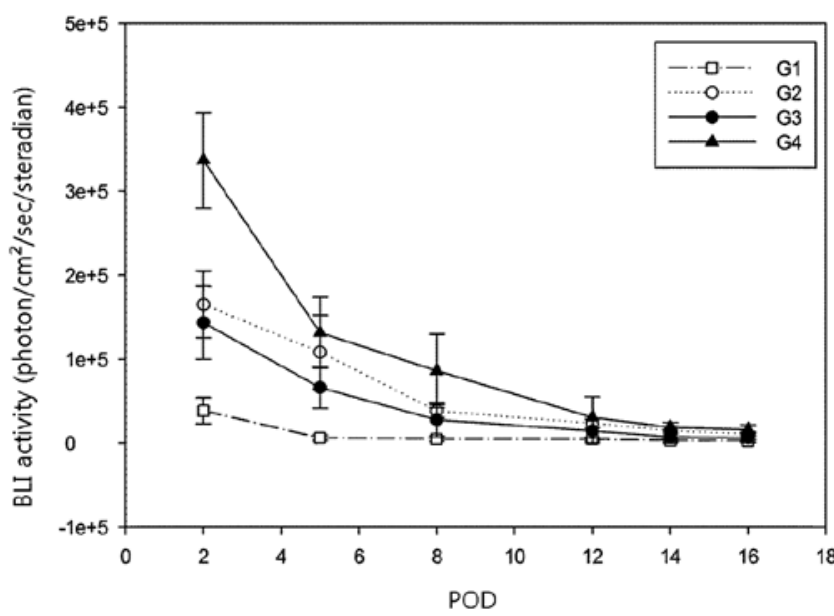

Figure 4. BLI revealed a decrease in activity over time in all study groups. BLI revealed continuous decreasing luciferase activity from the ADMSCs in G1 to G4. All groups revealed only a small amount of residual activity in the later stage of the experiment. There was no increment of BLI activity to suggest the proliferation of ADMSCs during the monitoring period.

volume of xenografted tumors on the right and left sides from all 4 groups because of the wide range of standard deviations. Thus, the tumor volume ratio of the AMDSC-treated side to the control side was introduced to reduce the bias from individual variance and this value showed significant differences between G2 and G4, and G3 and G4, by the Mann-Whitney $\mathrm{U}$ test (Fig. 3). However, the Pearson correlation coefficient between the last tumor volume and initial BLI activity in G2 and G3 was 0.453 , which showed no significant correlation between these two factors $(\mathrm{P}=0.1915)$.

Proliferation and survival of ADMSCs in the xenografts. Initial BLI revealed strong luciferase activity from the ADMSCs in all xenografts, but these activities rapidly decreased at an early stage, and only minimal residual luciferase activities were detected during the later stages. There was no definite increment in BLI to suggest significant proliferation of ADMSCs during the follow-up period and no BLI activity was detected in contralateral tumors to suggest distant migration of the stem cells (Fig. 4) 


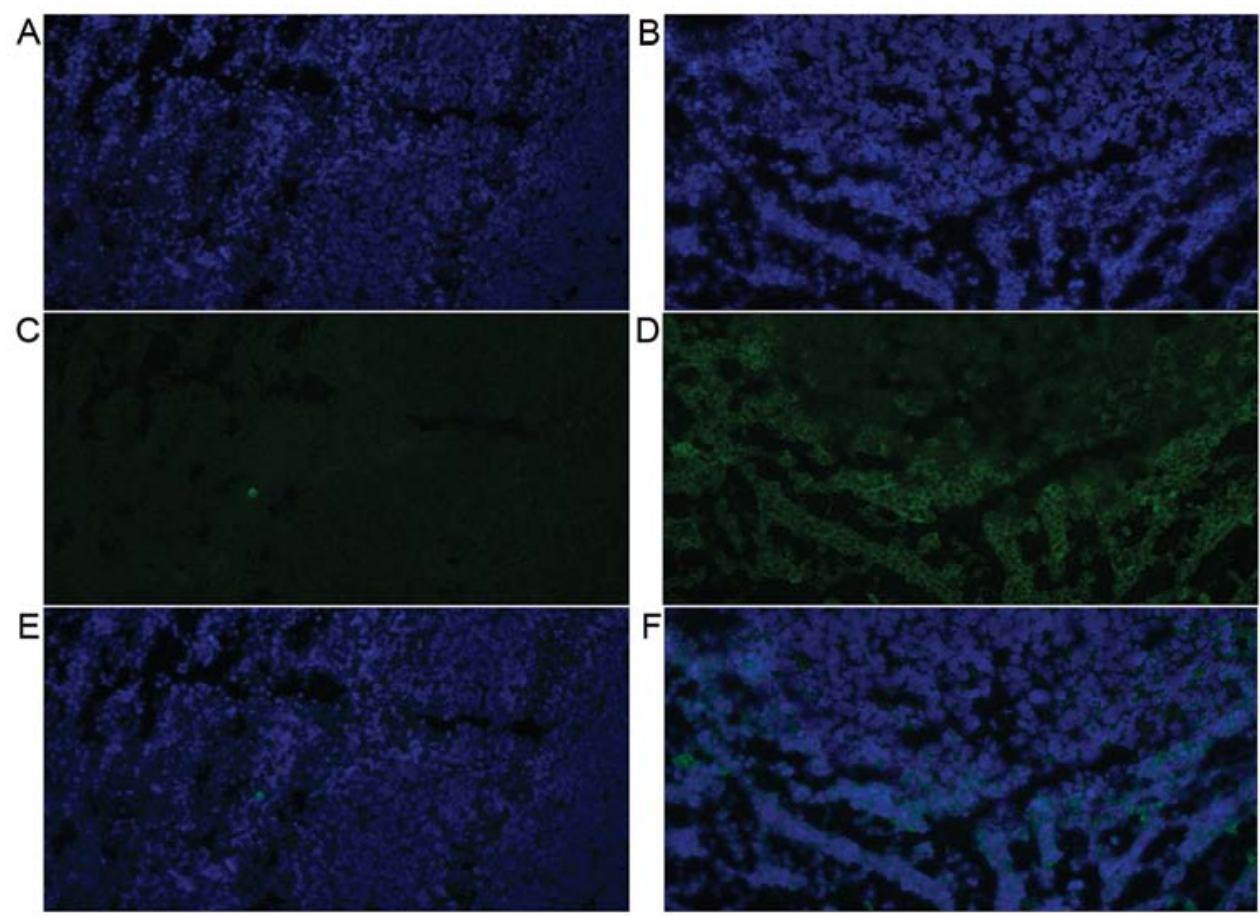

Figure 5. Immunohistochemical analysis of control and ADMSC-treated tumors. DAPI staining of the control (A) and ADMSC-treated sides (B) revealed similar findings. Immunohistochemical analysis of luciferase activity showed no activity in the control tumor (C). Only a small number of stained cells were found in the periphery of the ADMSC-treated tumor mass (D). The fusion of DAPI and luciferase staining of the control (E) and ADMSC-treated sides (F) was added for a better understanding of ADMSC distribution in the tumor specimen.

Histological analysis. The DAPI staining of both the control side and ADMSC-treated side revealed well stained nuclei (Fig. 5A and B) but immunohistochemical staining for luciferase in the control side showed no positive finding to suggest any migration of AMDSCs from the primary administration site (Fig. 5C). Fluorescent microscopic images of the ADMSC-treated side displayed a few bright cells stained by the luciferase antibody at the periphery of the tumor mass (Fig. 5D). However, these cells were not detected at the center of the mass, and there was no visible distribution of stained cells along the vascular structures or interstitial tissues, which might have suggested the contribution of ADMSCs to these components.

\section{Discussion}

Stem cells are a special type of cells characterized by self-renewal and pluripotency. They can be classified into embryonic stem cells (ESCs) and adult stem cells by their origin. ESCs are ideal, considering their differentiation ability and capacity for self-renewal, but practical and ethical issues prevent their widespread and active clinical application (3). Therefore, adult stem cells are emerging as an alternative to ESCs due to their accessibility and wide applicability in clinical and research fields (19). The known sources of adult stem cells are bone marrow $(10,14)$, fat tissue $(20)$, peritoneum and synovium (21).

Among the adult stem cells, MSCs originating from bone marrow were initially cultured and investigated (22). The subsequent identification of adipose tissues as a source of MSCs has gained much attention, as it presents an alternative source from which these cells could be harvested more easily and in greater numbers (20). However, the characteristics of ADMSCs and BMMSCs have not been fully defined, and data describing the effects of these cells on tumor growth have been contradictory (23). Several studies have described the contribution of BMMSCs to tumor neovasculature (14) or tumor cell growth in vivo $(11,24)$. Most studies on the inhibitory effect of MSCs on tumorigenesis were also performed using BMMSCs (7-9,25,26). Only one study addressed this issue using ADMSCs, and its analyses were performed in vitro (18). This study was the starting point for the present study. Although there have been several reports concerning the trafficking of stem cells (27) and therapeutic delivery vehicles targeting tumor stroma (5), the proportion of stem cells migrating to target organs is very small when using intravenous administration (28). Therefore, it has been suggested that the clinical application of MSCs should be handled with extreme caution, especially in malignant disease (23).

In the present study, we aimed to evaluate the effect of the local treatment of ADMSCs in a xenografted animal model using BLI and micro-US. In the first trial, two proportions of ADMSCs were used at G1 (5\%) and G3 (15\%), and despite the same number of UMRs being inoculated into the control side (left), the final volume of tumors without ADMSCs was significantly different between G1 $\left(38.06 \mathrm{~mm}^{3}\right)$ and $\mathrm{G} 3$ $\left(127.56 \mathrm{~mm}^{3}\right)$. The effect of ADMSCs on tumorigenesis in G1 must be underestimated due to the abnormally small tumor volume on the control side. The comparison of the tumor volumes on the ADMSC-treated and control sides of the mice revealed tumor suppression in $4 / 5$ mice in G3, but only $2 / 5$ mice in G1. This was despite the average volume of the 
ADMSC-treated side in G1 $\left(72.9 \mathrm{~mm}^{3}\right)$ and G2 $\left(93.39 \mathrm{~mm}^{3}\right)$ showing no statistically significant difference. This result might be attributed to the poor condition of the frozen UMR stock used in this experiment. In the second trial, markedly different results were noted between mice in $\mathrm{G} 2$ and $\mathrm{G} 4$, which were injected with UMRs containing 10 and 25\% ADMSCs, respectively. G2 showed results similar to those of G3, with 4/5 mice exhibiting a smaller tumor volume on the ADMSCtreated side. However, no mice in G4 showed a smaller volume on the treated side than the control side, and the ADMSCtreated side showed marked acceleration of tumor growth as summarized in Table II. Similar results were also observed in the cases where tumor suppression failed in G2 and G3, although G1 data could not be used to assess this finding due to the unreliable volume data from the control side. The differences in the tumor volume ratios between G2 vs. G4 and G3 vs. G4 for the estimation of the tumor-suppressive effect were confirmed by Mann-Whitney U test $(\mathrm{P}=0.0159)$.

Luciferase activity measured by BLI revealed a continuously decreasing pattern, suggesting a lack of proliferation of ADMSCs in the tumor masses, although the decrement rate was different among the groups. This was also confirmed by immunohistochemical staining for luciferase in the tumor specimens after sacrificing the animals. Immunohistochemical analysis of ADMSCs using an anti-luciferase antibody revealed only a small number of fluorescence signals in the periphery of the tumor mass. No significant activity was detected along the vascular structure or interstitium of the tumor. According to the results of BLI and immunohistochemistry, we suggest that most of the locally administered ADMSCs perished within the tumor mass without evidence of proliferation or contribution to the source of the stromal structures of the tumors.

According to our results, locally administered AMDSCs appear to act as a tumor suppressor to a certain degree at low proportions, whereas higher proportions of ADMSCs act as a tumor stimulator. Considering BLI and the immunohistochemistry findings, these effects of ADMSCs appear to be more likely via early humoral effects such as the production of various cytokines and excretions as previously described $(9,29,30)$, rather than by direct effects from the continuous proliferation of the stem cells themselves. An additional noteworthy finding is that for two tumors in G2 and G3 where there was a failing ADMSC-mediated suppressive effect, there was very rapid proliferation and this finding may suggest that the tumor-suppressive effect might be determined at an early stage.

Our study has several limitations. First, the small size of the studied population is a major limitation, which resulted from limitations in the numbers of animals that can be imaged at one time point in the BLI facility, and the numbers of ADMSCs obtained from a single batch. Therefore, a statistically significant result could not be obtained when comparing the mean volumes of tumors with and without ADMSCs. Nevertheless, we suggest that this experiment may be useful as a pilot study to act as a platform for future experiments. The second limitation is that UMRs showed heterogeneous growth rates in the tumor xenografts despite the same number of cells being inoculated, resulting in difficulties in the interpretation of G1 data. The final limitation is that in vivo BLI activity could not precisely identify reporter expression of ADMSCs, such as light propagation in different optical barriers such as hemorrhages or discoloration of tumor, or any individual variation in the metabolism of intraperitoneally administered luciferin.

However, despite all of these limitations, the present study provides information concerning the behaviors and actions of local treatment with ADMSCs in early tumorigenesis and the growing period, and further study using larger numbers of animals is necessary to elucidate the exact role of ADMSCs in tumor growth.

\section{References}

1. Thomson JA, Itskovitz-Eldor J, Shapiro SS, et al: Embryonic stem cell lines derived from human blastocysts. Science 282: 1145-1147, 1998

2. Wagner AM, Krenger W, Holzgreve W, Burkle P and Surbek DV: Use of human embryonic stem cells and umbilical cord blood stem cells for research and therapy: a prospective survey among health care professionals and patients in Switzerland. Transfusion 53: 2681-2689, 2013.

3. Faulkner SD, Vawda R and Fehlings MG: Adult-derived pluripotent stem cells. World Neurosurg 82: 500-508, 2014.

4. Cao F, Lin S, Xie X, et al: In vivo visualization of embryonic stem cell survival, proliferation, and migration after cardiac delivery. Circulation 113: 1005-1014, 2006.

5. Serakinci N, Christensen R, Fahrioglu U, et al: Mesenchymal stem cells as therapeutic delivery vehicles targeting tumor stroma. Cancer Biother Radiopharm 26: 767-773, 2011.

6. Nakamizo A, Marini F, Amano T, et al: Human bone marrowderived mesenchymal stem cells in the treatment of gliomas. Cancer Res 65: 3307-3318, 2005.

7. Khakoo AY, Pati S, Anderson SA, et al: Human mesenchymal stem cells exert potent antitumorigenic effects in a model of Kaposi's sarcoma. J Exp Med 203: 1235-1247, 2006.

8. Qiao L, Xu Z, Zhao T, et al: Suppression of tumorigenesis by human mesenchymal stem cells in a hepatoma model. Cell Res 18: 500-507, 2008.

9. Katsuno T, Ochi M, Tominaga K, et al: Mesenchymal stem cells administered in the early phase of tumorigenesis inhibit colorectal tumor development in rats. J Clin Biochem Nutr 53: 170-175, 2013.

10. Studeny M, Marini FC, Champlin RE, Zompetta C, Fidler IJ and Andreeff M: Bone marrow-derived mesenchymal stem cells as vehicles for interferon-beta delivery into tumors. Cancer Res 62: 3603-3608, 2002.

11. Zhu W, Xu W, Jiang R, et al: Mesenchymal stem cells derived from bone marrow favor tumor cell growth in vivo. Exp Mol Pathol 80: 267-274, 2006.

12. Djouad F, Plence P, Bony C, et al: Immunosuppressive effect of mesenchymal stem cells favors tumor growth in allogeneic animals. Blood 102: 3837-3844, 2003.

13. Studeny M, Marini FC, Dembinski JL, et al: Mesenchymal stem cells: potential precursors for tumor stroma and targeted-delivery vehicles for anticancer agents. J Natl Cancer Inst 96: 1593-1603, 2004.

14. Davidoff AM, Ng CY, Brown P, et al: Bone marrow-derived cells contribute to tumor neovasculature and, when modified to express an angiogenesis inhibitor, can restrict tumor growth in mice. Clin Cancer Res 7: 2870-2879, 2001.

15. Patel SA, Heinrich AC, Reddy BY, Srinivas B, Heidaran N and Rameshwar P: Breast cancer biology: the multifaceted roles of mesenchymal stem cells. J Oncol 2008: 425895, 2008.

16. Dwyer RM, Khan S, Barry FP, O'Brien T and Kerin MJ: Advances in mesenchymal stem cell-mediated gene therapy for cancer. Stem Cell Res Ther 1: 25, 2010.

17. Schäffler A and Büchler C: Concise review: adipose tissuederived stromal cells - basic and clinical implications for novel cell-based therapies. Stem Cells 25: 818-827, 2007.

18. Zhao W, Ren G, Zhang L, et al: Efficacy of mesenchymal stem cells derived from human adipose tissue in inhibition of hepatocellular carcinoma cells in vitro. Cancer Biother Radiopharm 27: 606-613, 2012.

19. Barry FP and Murphy JM: Mesenchymal stem cells: clinical applications and biological characterization. Int J Biochem Cell Biol 36: 568-584, 2004. 
20. Zuk PA,Zhu M, Ashjian P, et al: Human adipose tissue is a source of multipotent stem cells. Mol Biol Cell 13: 4279-4295, 2002.

21. Preston SL, Alison MR, Forbes SJ, Direkze NC, Poulsom R and Wright NA: The new stem cell biology: something for everyone. Mol Pathol 56: 86-96, 2003.

22. Lyden D, Hattori K, Dias S, et al: Impaired recruitment of bonemarrow-derived endothelial and hematopoietic precursor cells blocks tumor angiogenesis and growth. Nat Med 7: 1194-1201, 2001.

23. Ramasamy R, Lam EW, Soeiro I, Tisato V, Bonnet D and Dazzi F Mesenchymal stem cells inhibit proliferation and apoptosis of tumor cells: impact on in vivo tumor growth. Leukemia 21 : 304-310, 2007.

24. Sato T, Sakai T, Noguchi Y, Takita M, Hirakawa S and Ito A: Tumor-stromal cell contact promotes invasion of human uterine cervical carcinoma cells by augmenting the expression and activation of stromal matrix metalloproteinases. Gynecol Oncol 92: 47-56, 2004

25. Ohlsson LB, Varas L, Kjellman C, Edvardsen K and Lindvall M: Mesenchymal progenitor cell-mediated inhibition of tumor growth in vivo and in vitro in gelatin matrix. Exp Mol Pathol 75: 248-255, 2003.
26. Nakamura K, Ito Y, Kawano Y, et al: Antitumor effect of genetically engineered mesenchymal stem cells in a rat glioma model. Gene Ther 11: 1155-1164, 2004.

27. Wu GD, Nolta JA, Jin YS, et al: Migration of mesenchymal stem cells to heart allografts during chronic rejection. Transplantation 75: 679-685, 2003

28. Barbash IM, Chouraqui P, Baron J, et al: Systemic delivery of bone marrow-derived mesenchymal stem cells to the infarcted myocardium: feasibility, cell migration, and body distribution. Circulation 108: 863-868, 2003.

29. Serakinci N, Fahrioglu U and Christensen R: Mesenchymal stem cells, cancer challenges and new directions. Eur J Cancer 50: 1522-1530, 2014

30. Ghannam S, Bouffi C, Djouad F, Jorgensen C and Noel D: Immunosuppression by mesenchymal stem cells: mechanisms and clinical applications. Stem Cell Res Ther 1: 2, 2010. 